\title{
BRAIN STUDANTS: GAMES E REALIDADE VIRTUAL E MISTURADA COMO METODOLOGIA ATIVA NO ENSINO DE CIÊNCIAS PARA ALUNOS DO $6^{\circ}$ ANO DO ENSINO FUNDAMENTAL EM ARAGUATINS, TO
}

\author{
BRAIN STUDANTS: GAMES AND VIRTUAL AND MIXED REALITY AS AN \\ ACTIVE METHODOLOGY IN SCIENCE TEACHING FOR STUDENTS OF THE 6th \\ YEAR OF FUNDAMENTAL EDUCATION IN ARAGUATINS, TO
}

\author{
Mateus de Souza Miranda ${ }^{1}$; Maristela Tavares Gonçalves ${ }^{2}$
}

\section{RESUMO}

Este artigo trata das influências das práticas tecnológicas Realidades Virtual (VR) e Misturada (MR) no processo educacional de alunos do $6^{\circ}$ ano do Ensino Fundamental e sua ingerência na interdisciplinaridade. Buscando esclarecer a atuação dos jogos digitais, este trabalho objetiva utilizar os games como modelo para criação de uma prática diferenciada com foco no melhoramento de aprendizagem de alunos. Valendo-se de uma pesquisa quantiqualitativa e exploratória, foi aplicada uma avaliação de conhecimentos aos alunos, verificado qual o menor índice de conhecimento e compreensão do que foi trabalhado em aula anteriormente, montada uma Oficina para treinamento das ferramentas tecnológicas, finalizando com Torneio de Games. Comparando o método avaliativo tradicional com a avaliação tecnológica, sucedeu-se incomparável nível de curiosidade, criatividade e interesse. Na avaliação tecnológica, os alunos apresentaram domínio do conteúdo, com um número 35,3\% maior de respostas corretas quando comparado ao modelo tradicional de avaliação. A presente pesquisa dá contraste à visão negativa sobre a utilização de games e a dicotomia de que os dispositivos digitais não contribuem enquanto ferramenta de ensino; sobrelevando seu potencial desenvolvimento nas habilidades cognitivas durante a aprendizagem. Destarte a primordialidade de preparação dos educadores para o uso desses aparatos, igualmente a sua introdução as outras ferramentas disponíveis. Para isso, é necessário que haja apoio institucional, social e principalmente governamental com investimentos em estrutura e qualificação dos mesmos a fim de que seja possível desfrutar de uma educação de qualidade com alunos engajados e ativos no meio social a que pertencem.

Palavras-Chave: Educação. Ensino colaborativo. Inovação. Práticas tecnológicas.

\begin{abstract}
\footnotetext{
${ }^{1}$ Graduando em Licenciatura da Computação - IFTO, mateusmirandaa2@ hotmail.com

${ }^{2}$ Esp. em Ensino Superior de Química - IFTO, maristela.tg@ifto.edu.br
}

This article deals with the influences of Virtual Realities (VR) and Mixed Techniques (MR) technological practices in the educational process of 6th grade students and their interdisciplinarity. Seeking to clarify the performance of digital games, this work aims to use games as a model to create a differentiated practice focused on the improvement of student learning. Using a quantitative and exploratory research, an evaluation of knowledge was applied to the students, verified the lowest index of knowledge and understanding of what was worked in class previously, set up a workshop to train the technological tools, finalizing with Games Tournament . Comparing the traditional evaluative method with the technological evaluation, there was an incomparable level of curiosity, creativity and interest. In the technological evaluation, the students presented mastery of the content, with a $35.3 \%$ greater number of correct answers when compared to the traditional evaluation model. This research contrasts the negative view about the use of games and the dichotomy that digital devices do not contribute 
as a teaching tool; raising their potential development in cognitive skills during learning. Thus, the primordiality of the preparation of the educators for the use of these apparatuses, also its introduction the other available tools. For this, it is necessary that there be institutional, social and mainly governmental support with investments in structure and qualification of the same so that it is possible to enjoy a quality education with students engaged and active in the social environment to which they belong.

Keywords: Education. Collaborative teaching. Innovation. Technological practices.

\section{INTRODUÇÃO}

A ausência de recursos, espaços e suporte para aperfeiçoamento e práticas metodológicas inovadoras cercam a educação comedindo-a, de forma que alunos e educadores se tornem manietados em métodos pedagógicos tradicionais ineficazes no contexto atual; a era tecnológica.

Os jogos digitais e as Realidades Virtuais (VR) e Misturadas (MR) são um dos destaques dessa era, tomando espaço e criando possibilidades presentes no cotidiano infantojuvenil: os games apresentam recursos lúdicos e atraentes que retêm a atenção de seus usuários. A tecnologia VR apresenta uma interface computadorizada que simula interações em tempo real, por meio de vários sensores entre máquina e usuário, conquanto que, a Realidade Aumentada mantém o usuário no ambiente real e traz objetos virtuais que interagem em tempo e espaço.

Mas não é de hoje que os games afluem crescendo despercebidamente, moldando-se para as tecnologias móbiles e ganhando espaço nas mais diversas áreas. De acordo com Kirner e Kirner (2011), a caracterização de Realidade Virtual dá-se em 1963 nos Estados Unidos, apesar de no final dos anos 30 o termo já ser usado; conquanto a Realidade Aumentada surgiu na década de 60, mas só nos anos 80 apareceram os primeiros projetos com essa tecnologia.

Utilizando tecnologias VR e MR, os jogos digitais vêm sendo correlacionados entre educação e crianças desde 1997 com o Apple II®, segundo Albuquerque e Fialho (2009).

Os games despertam o interesse dos educandos por seu layout divertido e colorido, e sobretudo imersivo pelo uso de VR e MR, contribuindo para uma melhor fixação do conteúdo teórico. Esta linha de pensamento é corroborada por Moran (2015), que enfatiza o uso de tecnologias e ensino híbrido através de jogos digitais e outras formas, que proporcionem a comunicação entre o mundo e a escola, pluralmente.

Por outro lado, Ribeiro (2009) afirma que os jogos digitais nem sempre se configuram como um exercício de ensino significativo, ou seja, ele não substitui os elementos fundamentais pedagógicos como os livros, nem a parte teórica e o debate provocado pelo professor durante as aulas.

Tendo em vista os aspectos observados, gerou-se a seguinte problemática: os games 
podem influenciar no processo educacional de alunos do sexto ano ( $6^{\circ}$ ano) do Ensino Fundamental, de modo à contribuir para as necessidades pedagógicas, complementando aprendizagem teórica, promovendo inclusão dos educandos e, amenizando assim, o(s) deficit(s) de ensino?

Para responder a esta questão, a pesquisa teve como objetivo utilizar os games como modelo para criação de uma prática diferenciada com foco no melhoramento de aprendizagem do grupo de alunos. Através de uma pesquisa quantiqualitativa e exploratória, foi identificada e avaliada qual a principal dificuldade dos alunos em uma disciplina, fomentando a prática e uso de métodos avaliativos ativos.

Concluiu-se que a presente pesquisa, contrasta a visão negativa sobre a utilização de games e a dicotomia que os dispositivos digitais não contribuem enquanto ferramenta de ensino, acentuando positivamente seu potencial de desenvolvimento das habilidades durante a aprendizagem.

\section{FUNDAMENTAÇÃO TEÓRICA}

Os jogos digitais são um dos destaques da era tecnológica, tomando espaço e criando possibilidades presentes no cotidiano infantojuvenil: usados como ferramenta educacional, os games apresentam recursos lúdicos e atraentes que retêm a atenção de seus usuários.

Aliado como um agente inovador à educação, os games podem cumprir positivamente seu papel de solucionar problemas educacionais existentes, transformando gradualmente as práticas docentes e mudando os métodos de ensino aprendizado ainda tradicionais. Aprender não significa ler e fazer exercícios em livros didáticos somente, mas ter novas experiências, e os jogos podem ser usados como simuladores e facilitadores do aprendizado, tese ratificada por diversos estudiosos que reforçam a ideia do uso de tecnologias digitais na educação:

[...] para aprender a dirigir um carro, não basta ler muito sobre esse tema; tem que experimentar, rodar com o ele em diversas situações com supervisão, para depois poder assumir o comando do veículo sem riscos. (MORAN, 2015, p.17)

Felício e Morais (2017) destacam que a inserção de tecnologias às escolas, não só proporciona mais qualidade à educação, como também o acesso à informação, convívio e inúmeras formas de aprender e ensinar. Albuquerque e Fialho (2009) reiteram este pensamento afirmando que essas ferramentas exigem esforços de seus jogadores no sentido de interação, envolvendo-os no âmbito, para que se sintam imersos na realidade artificial. 
Os jogos eletrônicos - educativos ou não - envolvem um processo de aprendizagem. Seus desafios crescentes, sua variedade de desafios e o dinamismo deste mercado, lançando novos jogos com grande velocidade, garantem que no mínimo, o jogo tenha que ser aprendido ao ser jogado pela primeira vez. (ALBUQUERQUE e FIALHO, 2009, p. 2)

Seguindo essa mesma visão, Wunsch (2017) diz que a VR, num contexto pedagógico, enriquece as metodologias trabalhadas em sala e imerge os educandos às temáticas. A autora questiona ainda acerca das fronteiras de aplicação dessa tecnologia no contexto escolar, concluindo dessa forma, que é necessário explorar a área científica e realizar manipulação de objetos nesse meio virtual a fim de tornar o ensino uma experiência completa e interativa.

Além disso, o uso de jogos digitais com gadgets auxiliadores como a Realidade Virtual (VR) e a Misturada (MR) também apresentam ingerência em outros aspectos positivos, como por exemplo, a interdisciplinaridade e o dinamismo; em contrapartida, há os aspectos negativos, como desconcentração no foco de estudo ou o vício, sendo portanto, um instrumento que necessita de acompanhamentos de seus mediadores.

De acordo com Ribeiro (2009), os jogos digitais nem sempre se configuram como um exercício de ensino significativo, ou seja, ele não substitui os elementos fundamentais pedagógicos como os livros, nem a parte teórica e o debate provocado pelo professor durante as aulas. Portanto, os educandos podem utilizar os games como um extensor de ensino, plataforma de simulação ou recurso de prática da teoria trabalhada num período de aprendizado.

Contudo, para que a ação 'aprender com jogos' possa ser implantada, deve-se pensar o espaço de aula de forma a dar suporte e liberdade aos alunos para usarem dispositivos tecnológicos, obtendo desse jeito, aproveitamento e rendimento do conteúdo aplicado, de acordo com Moran (2015).

Nesse sentido, o autor contribui para a ideia de integração pois, a escola para se valer deste método de ensino, tem que se apresentar multifuncional possibilitando a execução das atividades.

\footnotetext{
O espaço virtual aproxima diversas realidades para uma só: o ambiente de ensino. $\mathrm{O}$ universo virtual integra espaços e tempos diferentes, o aprendizado se torna constante, ampliando o conhecimento além da sala de aula, por exemplo [...]. O educador não precisa se comunicar só frente a frente com os alunos, mas de diversas maneiras que o contexto exija. (MIRANDA, et al. 2017, p. 4)
}

Os games farão parte deste processo de multifuncionalidade que consiste na comunicação, aprendizagem, diversão e ativação do eu aluno enquanto colaborador social. Nesta linha de raciocínio, aplicativos/games voltados ao público com deficiência auditiva por exemplo, podem ser utilizados por toda a classe, promovendo a inclusão.

Destaca-se, que educadores devem estar em posição de mediadores neste processo, 
sendo agentes transformadores através de metodologias ativas (MORAN, 2015). E para tal, os professores necessitarão de formação continuada para usufruir e explorar as funcionalidades e recursos oferecidos pelos games, já que "para serem utilizados como instrumentos educacionais, eles devem conter características vinculadas à aprendizagem, possuir objetivos pedagógicos, sua utilização deve estar inserida em um contexto e em uma situação de ensino, baseados em uma metodologia" (CICHELERO, 2014, p. 24).

Em virtude do que foi exposto, pode-se afirmar que os recursos digitais contribuem para o fortalecimento do conteúdo teórico, das habilidades cognitivas dos alunos, a concentração e o raciocínio lógico, promovendo o acesso à informação de modo divertido. Esses recursos fomentam o aprendizado, dando ao educador um papel mais dinâmico e valorizando seus saberes.

\section{METODOLOGIA}

Baseando-se nas teorias de Moran (2015) e adaptando para a realidade escolar da região, a pesquisa foi planejada de forma a cunhar resultados quantiqualitativo, analisar, executar e reavaliar o uso de realidade virtual como método de ensino aprendizagem.

Para tanto, foi realizada a aplicação de uma avaliação geral de conhecimentos dos alunos. De posse dos dados obtidos nessa avaliação, foi verificada qual disciplina obteve o menor índice de conhecimento e compreensão do que foi trabalhado em aula anteriormente, baseando nestes resultados, a montagem de Oficina para instrução no uso das tecnologias. Estando os alunos aptos a utilizar as ferramentas, realizou-se um Torneio de Games.

Finalizando o Projeto de Pesquisa, os resultados foram tabulados, analisados e disponibilizados à Escola e seus educadores.

O período para a aplicação da pesquisa foi de dois meses, na escola Arte de Crescer, Araguatins-TO, tendo como público-alvo 17 alunos do $6^{\circ}$ ano vespertino do ensino fundamental II e o envolvimento de duas educadoras, uma professora de ciências e a coordenadora do macroprojeto de extensão.

Ressalta-se que, três alunos da classe selecionada para a pesquisa, atuaram como coorientadores do Projeto, visando apresentar o uso de metodologia científica na busca de soluções para a problemática apresentada.

\section{Aplicação do Projeto de Pesquisa}




\section{Avaliação de Conhecimento Geral}

O primeiro passo consistiu na elaboração e seleção de questionários e aplicação dos mesmos, para avaliação do conhecimento geral, contendo 25 questões objetivas e subjetivas.

Figura 1: Alunos participantes do Projeto elaborando a avaliação de conhecimento geral

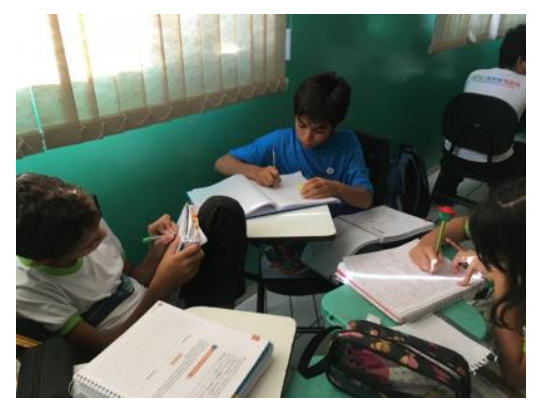

Fonte: Miranda, M. 2018.

Para a confecção dos questionários avaliativos, foram utilizados livros didáticos da própria escola, classificando as questões de acordo com as áreas de conhecimento (Ciências Exatas, Ciências da Natureza, Linguagens, Ciências Humanas e Ciências Biológicas), abrangendo as disciplinas de Matemática, Língua Portuguesa e Ciências como áreas mais exigentes para pontuação.

A aplicação da avaliação ocorreu em um único dia, com duração de 1h15min (uma hora e quinze minutos). Nesse dia ocorreu um contratempo: devido aos jogos escolares estarem em pleno acontecimento, não foi possível que todos os quatorze alunos participassem da avaliação inicial, somente doze foram avaliados.

Figura 2: Aplicação da avaliação sobre o nível de conhecimento geral

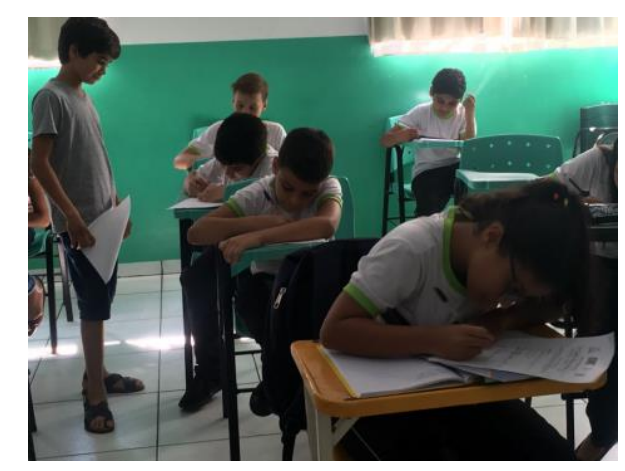

Fonte: Miranda, M. 2018.

Diante deste percalço, em reunião com a equipe, decidiu-se não marcar uma outra data para finalizar o exame, partindo do pressuposto que os alunos ausentes poderiam ser tentados a 
burlar a avaliação através do conhecimento prévio das questões apresentadas.

Não havendo tempo disponível para um novo questionário avaliativo, mantendo o rigor científico e ético da Pesquisa, decidiu-se avaliar as questões respondidas e levar em consideração a área em que os alunos apresentaram dificuldade na compreensão.

\section{Oficina para conhecimento das tecnologias de Realidade Virtual}

A Oficina ocorreu no Instituto Federal do Tocantins, campus Araguatins que disponibilizou um laboratório Interdisciplinar de Informática com toda a estrutura tecnológica necessária para tal.

Os alunos coorientadores fizeram, no primeiro momento, apresentação das ferramentas empregadas na Oficina: os games, Realidade Virtual (VR) e Realidade Misturada (MR). Num segundo momento, foi repassado o ensino de Ciências (Solos, devastações, Queimadas, Desmatamentos, o gerenciamento de Lixo) operando as tecnologias explanadas e finalizando com a realização de um debate sobre as temáticas abordadas.

Os aplicativos utilizados foram:

Expedições - Google ${ }^{\circledR}$ : uso guiado em VR;

Discovery VR®: uso em VR;

Youtube ${ }^{\circledR}$ : vídeos de gerenciamento de lixo em VR;

Minecraft $\AA$ : uso em ciências do solo;

Fitness AR®: uso em MR para demonstração dos tipos de relevos de solo;

* Anatomia Humana®: uso em MR para demonstração do sistema respiratório.

Figura 3: Oficina sobre das ferramentas de Realidade Virtual

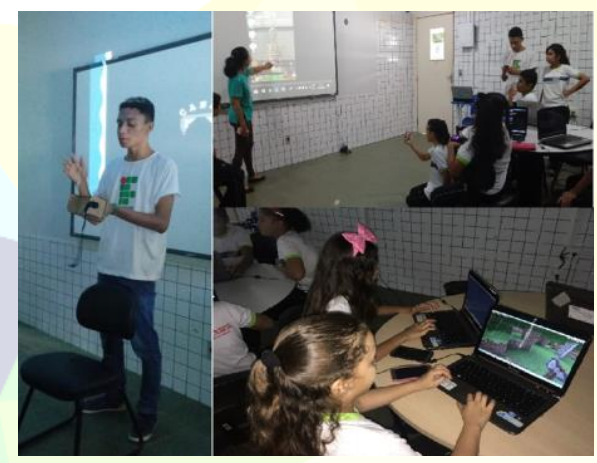

Fonte: Miranda, M. 2018.

Validando as teorias dos estudiosos de que o uso dos games deve ser um aliado às aulas teóricas, a professora de Ciências da classe foi convidada a participar da Oficina, contribuindo 
para o fortalecimento dos conteúdos teóricos aplicados em sala de aula e auxiliando a equipe de alunos no desenvolvimento das atividades.

\section{Torneio de Games}

Modelado na metodologia de Moran (2015), a classe foi dividida em três equipes, sendo duas equipes com 5 alunos e, uma equipe com quatro alunos. Trabalhando a interdisciplinaridade, foi feito o sorteio dos alunos para a divisão das equipes que se prepararam previamente, nomeando suas equipes, criando gritos de guerra e vestimentas que os caracterizassem durante o torneio.

As provas do Torneio de games foram:

* Caça Minério - as equipes procuraram ferro e ouro, no menor tempo possível, utilizando como ferramenta o game Minecraft $\AA$;

* Astronauta - através do uso de realidade virtual, as equipes identificaram os planetas pelo quesito 'aparência do solo', usando como meio de comunicação a mímica;

* Quiz - Usando o aplicativo Kahoot® tendo como objetivo responder as questões de ciências.

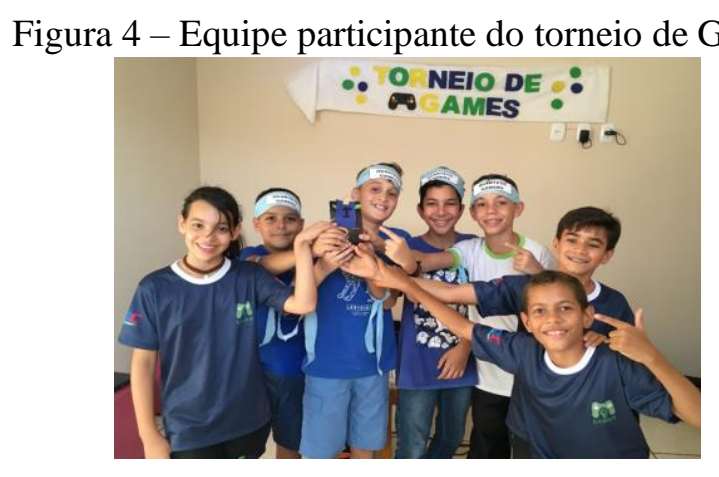

Fonte: Miranda, M. 2018.

A equipe vencedora foi a que concluiu todas as provas em menor tempo tendo a maior pontuação nos jogos.

O Torneio de Games significou para os alunos uma avaliação do conteúdo ministrado em aula de forma lúdica, conquanto que, para o Projeto, teve o propósito qualitativo de examinar o nível de fixação do conteúdo através da utilização das ferramentas tecnológicas.

\section{RESULTADOS E DISCUSSÃO}

Após o levantamento inicial, realizado através da aplicação de uma avaliação tradicional, firmou-se que a disciplina à ser trabalhada seria de ciências devido elevada 
porcentagem de respostas erradas, além de, concomitante a este projeto, realizar a apresentação dos resultados na Mostra de Pesquisa Científica da Escola Arte de Crescer - a MPEC 2018. Desta forma, foi trabalhada mais uma vez, a interdisciplinaridade.

\section{Avaliação Tradicional versus Torneio de Games}

A primeira avaliação de conhecimento geral, feita no modo tradicional através de um questionário escrito, teve um expressivo desinteresse por parte dos alunos em responder as questões de ciências. No entanto, houve uma alta taxa de acerto de questões de matemática.

O Gráfico 1 apresenta a quantidade de questões respondidas, as não respondidas e a de acertos do total de 12 alunos que participaram da avaliação tradicional. A quantidade de acertos máxima seria de 60 em matemática, e em ciências 36 - consideramos assim estes valores como $100 \%$ de cada disciplina.

Gráfico 1 - Comparativo do total de acertos nas avaliações das disciplinas de matemática e Ciências na avaliação tradicional

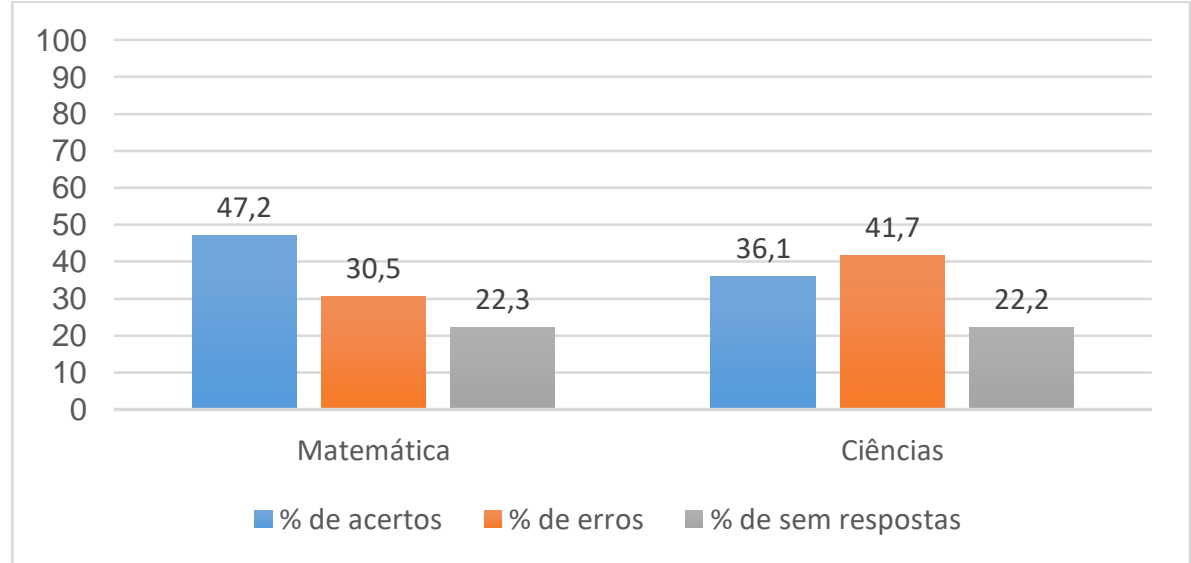

Fonte: Miranda, M. 2018.

O resultado dessa avaliação tradicional foi de $47,2 \%$ de acertos contra $30,5 \%$ de respostas erradas e $22,3 \%$ de questões sem respostas na disciplina de matemática; $36,1 \%$ de acertos, $41,7 \%$ de respostas incorretas e $22,2 \%$ sem respostas em ciências - tendo um número tão baixo de acertos, a disciplina de ciências foi a escolhida para uma nova avaliação usando as tecnologias.

Comparando o método avaliativo tradicional com a avaliação tecnológica denominada Torneio de Games, sucedeu-se um incomparavelmente nível de curiosidade, criatividade e sobretudo, interesse em participar da avaliação de forma espontânea, perceptível nos resultados: nenhuma alternativa ficou sem resposta. 


\section{MIRANDA, GONÇALVES}

Gráfico 2 - Comparativo do total de acertos nas avaliações das disciplinas de matemática e Ciências na avaliação tecnológica

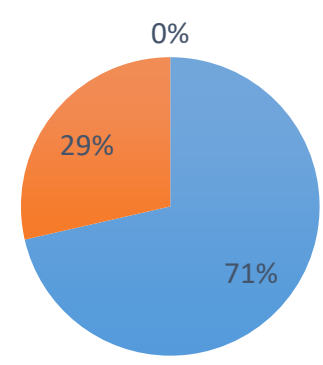

\% de acertos $\quad \%$ de erros $\quad \%$ sem respostas

Fonte: Miranda, M. 2018.

$\mathrm{Na}$ avaliação tecnológica, os alunos apresentaram domínio do conteúdo, com um número 35,3\% maior de respostas corretas quando comparado ao modelo tradicional; além da avaliação do conhecimento adquirido em sala de aula pelos alunos, foi possível neste segundo momento aceirar os pontos que ficaram escusos.

Num terceiro momento, foi realizado um debate entre os alunos e a equipe evidenciando que o método avaliativo tradicional causa desinteresse por sua habitualidade, tanto como qualquer outro exame escolar padrão.

Notabiliza-se que os alunos apresentaram domínio no uso de games com VR e MR durante o Torneio de games, sendo visível a facilidade de assimilação dos discentes quanto às tecnologias disponíveis.

\section{CONCLUSÕES}

Aprender tornou-se uma prática divertida e sem a percepção que se está aprendendo como nas metodologias tradicionais, o que cria um bloqueio mental, dificultando a compreensão e assimilação do conteúdo.

Os games e o uso de VR e MR para o ensino a alunos do Fundamental se efetivam, especialmente, no campo de conhecimento fugindo da tendência tradicionalista, ainda impregnada em quase todas as escolas do país, possibilitando seu uso interdisciplinar.

O aluno torna-se um ator no processo e no produto do ensino, passando a ser ativo e transformando o relacionamento professor-aluno e aluno-conteúdo.

A presente pesquisa dá contraste a visão negativa sobre a utilização de games e a dicotomia de que os dispositivos digitais não contribuem enquanto ferramenta de ensino; sobrelevando seu potencial desenvolvimento nas habilidades cognitivas durante a aprendizagem. Conseguinte, essas ferramentas se mostram necessárias e eficazes ao contexto 
atual da educação, empenhando papel motivador, lúdico e instigador, além de contribuir para o pensamento lógico.

Por outro lado, aponta-se a dispersão dos educandos durante o uso dessas tecnologias, sendo necessário acompanhamento do professor para evitar o desvio de atenção à temática que é trabalhada em sala.

Destarte, a primordialidade de preparação dos educadores para o uso desses aparatos, igualmente a sua introdução às outras ferramentas disponíveis que também podem proporcionam qualidade no ensino. Para isso, é necessário que haja apoio institucional, social e principalmente governamental com investimentos em estrutura e qualificação dos mesmos.

Com professores motivados é possível despertar ainda mais vocações e inovação no que concerne aos alunos, para que no futuro seja possível desfrutar de uma educação de qualidade com alunos engajados e ativos no meio social a quem pertencem.

\section{REFERÊNCIAS}

ALBUQUERQUE, R.; FIALHO, F. Concepção de jogos eletrônicos educativos: Proposta de processo baseado em dilemas. 2009. Rio de Janeiro (RJ). Disponível em: <http://www.sbgames.org/papers/sbgames09/artanddesign/60398.pdf $>$, Acesso em: 21 de abril de 2018.

CICHELERO. F. Um Serious Games para apoio a Pré-Alfabetização. 2014. Universidade de Caxias do Sul. Caxias do Sul. Disponível em: < https://repositorio.ucs.br/xmlui/bitstream/handle/11338/1366/TCC\%20Flavio\%20Cichelero.p

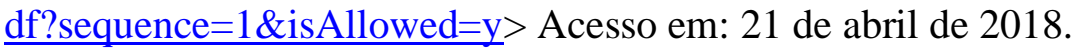

FELÍCIO, L.; MORAIS S. A Influência das novas Tecnologias nos aspectos psicomotores no Ensino Fundamental I. 2017. UnilaSalle, v. 9, n18. Niterói. Disponível em: $<$ https://revistas.unilasalle.edu.br/index.php/conhecimento_diversidade/article/view/4098/pdf $>$, Acesso em 21 de abril de 2018.

KINER, C; KINER, T. Evolução e Tendências da Realidade Virtual e da Realidade Aumentada. Sociedade Brasileira de Computação - SBC. XIII Symposium on Virtual and Augmented Reality. Uberlândia, MG. 2011.

Miranda, M. ,et al. As Tecnologias nas Práticas de Ensino-Apredizagem: Jovens Colaboradores. Instituto Federal do Tocantins. Araguatins, TO. 2017. Disponível em: < https://drive.google.com/file/d/1EejBsdzkEoTXHx97zRe91paVhSO98ZDX/view> Acesso em: 21 de abril de 2018.

MORAN, J. Mudando a educação com metodologias ativas. 2015. Coleção Mídias Contemporâneas. Convergências Midiáticas, Educação e Cidadania: aproximações jovens. Vol. II. São Paulo. Disponível em: < http://www2.eca.usp.br/moran/wpcontent/uploads/2013/12/mudando_moran.pdf > Acesso em: 23 de abril de 2018. 
RIBEIRO, F. Jogos e Modelagem na Educação Matemática. 2008, ed. 20. Curitiba. Disponível em:

$<$ https://books.google.com.br/books?id=vCAWYYnnxE8C\&printsec=frontcover\&dq=Jogos+ e+Modelagem+na+Educa\%C3\%A7\%C3\%A3o+Matem\%C3\%A1tica\&hl=pt-

$\mathrm{BR} \& \mathrm{sa}=\mathrm{X} \& \mathrm{ved}=0 \mathrm{ahUKEwiJ}$ bLCldTaAhXKDpAKHVdYAE0Q6AEIKDAA\#v=onepage \&q $=$ Jogos $\% 20 \mathrm{e} \% 20$ Modelagem $\% 20$ na $\% 20$ Educa $\% \mathrm{C} 3 \%$ A7\%C3\%A3o\%20Matem\%C3\%A1tica $\& \mathrm{f}=$ false $>$ Acesso em: 23 de abril de 2018.

WUNSCH, L.; RICHTER, A.; MACHADO, M. Realidade Virtual: Apoio para a prática contextualizada e interdisciplinar na Educação Básica. EDUCERE, ed, 6. Curitiba, PR. 2017. Disponível em: <http://educere.bruc.com.br/arquivo/pdf2017/24758_13541.pdf> Acesso em: 01 de maio de 2018. 